\title{
Overexpression of CENP-H as a novel prognostic biomarker for human hepatocellular carcinoma progression and patient survival
}

\author{
GUIFANG LU ${ }^{1}$, TAO SHAN ${ }^{2}$, SHUIXIANG HE ${ }^{1}$, MUDAN REN ${ }^{1}$, MENG ZHU ${ }^{1}$, \\ YANNAN HU ${ }^{1}$, XINLAN LU ${ }^{1}$ and DAN ZHANG ${ }^{1}$ \\ ${ }^{1}$ Department of Gastroenterology, First Affiliated Hospital of the Medical College, Xi'an Jiaotong University, \\ Xi'an, Shaanxi 710061; ${ }^{2}$ Department of General Surgery, Second Affiliated Hospital of Medical College, \\ Xi'an Jiaotong University, Xi'an, Shaanxi 710004, P.R. China
}

Received May 19, 2013; Accepted July 9, 2013

DOI: $10.3892 /$ or.2013.2675

\begin{abstract}
Centromere protein H (CENP-H) has been shown to be significantly upregulated in many types of cancers and is associated with disrupted cell cycle regulation, cell proliferation and genetic instability. The aim of the present study was to explore the expression and localization of CENP-H in hepatocellular carcinoma (HCC) and determine whether its overexpression is a prognostic biomarker for HCC. Reverse transcription-polymerase chain reaction (PCR), real-time qPCR and western blotting were used to compare CENP-H expression at the mRNA and protein levels in HCC samples and corresponding adjacent non-cancerous samples. CENP-H protein levels were determined in 60 paired paraffinembedded HCC tissues using immunohistochemistry (IHC), and the correlation with clinicopathological features and patient prognosis was analyzed. In addition, an immunofluorescence assay was performed to test the expression and localization of CENP-H protein in HCC cells. Results showed that levels of CENP-H mRNA and protein were higher in $\mathrm{HCC}$ samples than in the corresponding adjacent non-cancerous samples. In 60 paired paraffin-embedded tissues, CENP-H was upregulated in the HCC samples $(38 / 60,63.3 \%)$ relative to the adjacent non-cancerous samples $(21 / 60,35 \%, \mathrm{P}=0.003)$, and a higher level of upregulation was associated with tumor size $(\mathrm{P}=0.032)$; higher histological grade $(\mathrm{P}=0.001)$; more advanced TNM stage $(\mathrm{P}=0.002)$ and Chinese clinical stage $(\mathrm{P}=0.008)$; and poorer prognosis. In addition, consistent with the results of IHC, the immunofluorescence assay showed that CENP-H was localized in the nucleus of Hep3B cells. CENP-H was overexpressed in $\mathrm{HCC}$, and its level of upregulation was
\end{abstract}

Correspondence to: Professor Shuixiang He, Department of Gastroenterology, First Affiliated Hospital of the Medical College, Xi'an Jiaotong University, 277 West Yanta Road, Xi'an, Shaanxi 710061, P.R. China

E-mail: hesx123@126.com

Key words: hepatocellular carcinoma, CENP-H, prognosis, biomarker an independent prognostic indicator, suggesting that CENP-H may be an effective therapeutic strategy for the treatment of HCC.

\section{Introduction}

Hepatocellular carcinoma (HCC) is one of the most common cancers and the third leading cause of cancer-related mortality worldwide. Its incidence and mortality rate in Asia are high and have also rapidly increased in the United States (1-3). Due to its characteristic fast growth and early metastasis, the overall 3-year survival rate of HCC patients after resection is 35-62\% and the 5-year survival rate is $17-50 \%$. Without treatment, the survival rate is $<10 \%$; with recurrence, it is $70 \%$ (4-7). HCC is one of the most aggressive and intractable malignant tumors and, unfortunately, the mechanisms contributing to its carcinogenesis and progression are still poorly understood.

Chromosomal instability (CIN) leading to aneuploidy is a characteristic feature of various types of cancer (8-11). Kinetochore, a structure composed of proteins such as centromere protein (CENP)-A, CENP-B, CENP-C, CENP-E, CENP-F, CENP-H and CENP-I/MIS6 (12-14), is assembled on centromeres on which spindle microtubules attach during mitosis to pull apart the sister chromatids. Normal expression of kinetochore is vital to mitosis (15); however, disruption of core centromere proteins may induce an increase in CIN and tumorigenesis (16). CENP-H, with an apparent molecular mass of $33 \mathrm{kDa}$, was first isolated from mouse and human CENP-H protein was shown to localize in the inner plate with CENP-A and CENP-C $(17,18)$. It is a fundamental component of the active centromere complex. Accumulating evidence indicates that CENP-H is frequently upregulated in cancers, and its overexpression and mislocation are associated with the development of aneuploidy, a hallmark of malignancy. Studies have shown that CENP-H is significantly associated with human nasopharyngeal carcinoma, esophageal carcinoma, tongue cancer, breast cancer, non-small cell lung cancer and gastric cancer (19-22). Increased expression of CENP-H increases the proliferative activity of human oral squamous cells (23), and depletion of CENP-H through RNA interference produces severe mitotic phenotypes such as misaligned chromosomes and multipolar spindles (24). Research has also revealed that 
Table I. The Chinese 2001 staging system.

Stage Description

Ia Signal tumor, the maximum diameter $\leq 3 \mathrm{~cm}$; no tumor thrombus; no intraperitoneal lymph node or distant metastasis; Child A.

Ib Signal or two tumors located in hemihepatis, the sum of maximum diameters $\leq 5 \mathrm{~cm}$; no thrombus; no intraperitoneal lymph node or distant metastasis; Child A.

IIa Signal or two tumors located in hemihepatis, the sum of maximum diameters $\leq 10 \mathrm{~cm}$, or the sum of maximum diameters of two tumors $\leq 5 \mathrm{~cm}$ located in the left and right half of liver respectively; no thrombus; no intraperitoneal lymph node or distant metastasis; Child A.

IIb Signal or two tumors located in hemihepatis, the sum of maximum diameters $>10 \mathrm{~cm}$, or the sum of maximum diameters of two tumors $>5 \mathrm{~cm}$ located in the left and right half of liver respectively, or more than two tumors; no thrombus; no intraperitoneal lymph node or distant metastasis; Child A.

Regardless of the tumors; there is thrombus of branch of portal vein, hepatic vein, or bile duct; no intraperitoneal lymph node or distant metastasis; Child A.

Regardless of the tumors; no thrombus; no intraperitoneal lymph node or distant metastasis; Child B.

IIIa Regardless of the tumors; there is one of the next two cases: thrombus of main portal vein or inferior vena cava, metastasis of intraperitoneal lymph node or distant metastasis; Child A/B.

$\mathrm{IIIb}$ Whatever the others; Child C.

CENP-H is closely associated with colorectal cancer and that its overexpression induces aneuploidy (25).

In the present study, we first confirmed the differential expression of CENP-H in 10 pairs of HCC samples and corresponding adjacent non-cancerous samples using reverse transcription-polymerase chain reaction (RT-PCR), real-time quantitative PCR (qPCR) and western blotting. We used immunohistochemistry to compare the expression of CENP-H with clinicopathological features and overall survival of patients with HCC. Finally, an immunoflurorescence assay was used to detect and localize the expression of CENP-H in HCC cells.

\section{Materials and methods}

Patient specimens. From January 2009 to December 2010, after obtaining approval from the Human Subjects Committee of the First Affiliated Hospital of Xi'an Jiaotong University and informed consent of the patients, HCC tissues (including adequately sized tumor tissue samples and the corresponding adjacent non-cancerous tissue samples obtained $5-10 \mathrm{~cm}$ from the tumor) were obtained from patients undergoing liver resection for hepatic cancer at the Department of Hepatobiliary Surgery, First Affiliated Hospital of Xi'an Jiaotong University. All tissues were obtained within 30 min following excision from the patient's body. A part of the sample was cut into small pieces, snap-frozen in liquid nitrogen immediately and stored at $-80^{\circ} \mathrm{C}$ to prepare for analysis of CENP-H gene expression, the other part was fixed with $4 \%$ formalin and paraffin-embedded for histological studies. Of the 60 patients, there were 49 men and 11 women. Median age at the time of surgery was 52 years (range, 25-74 years). The histological type in all 60 patients was hepatocellular carcinoma, diagnosed according to the World Health Organization histological classification of tumors of the liver and intrahepatic bile ducts (2000) (26). The disease stages of all the patients were classified or reclassified according to the classification of the International Union Against Cancer (27) and the Chinese Anti-Cancer Association, as outlined in Table I. Clinical information of the samples is described in detail in Table II. Patients with preoperative anticancer treatment or with evidence of other malignancies were excluded from the study. All patients were followed up, and the median duration of follow-up was 20 months (range, 2-39 months).

RNA isolation, RT-PCR and real-time $q P C R$. Total RNA was extracted from HCC tumors and the corresponding non-tumor tissues with TRIzol reagent (Invitrogen, Carlsbad, CA, USA) according to the manufacturer's instructions. First-strand cDNA was synthesized from total RNA with PrimeScript ${ }^{\circledR}$ RT Master Mix [Takara Biotechnology (Dalian) Co., Ltd., Dalian, China].

The PCR primers sets were as follows: i) CENP-H, forward 5'-CAGTCTAGTGTGCTCATGGAT-3' and reverse 5'-TCCA TCTGTAGGTTTTGTCG-3'; ii) glyceraldehyde-3-phosphate dehydrogenase (GAPDH), forward 5'-CAAGCTCATTTCC TGGTATGAC-3' and reverse 5'-CAGTGAGGGTCTCTCTC TTCCT-3'. The RT-PCR conditions included an initial denaturation step for $5 \mathrm{~min}$ at $94^{\circ} \mathrm{C}$ followed by 30 cycles of amplification: $94^{\circ} \mathrm{C}$ for $30 \mathrm{sec}, 55^{\circ} \mathrm{C}$ for $30 \mathrm{sec}$ and $72^{\circ} \mathrm{C}$ for $30 \mathrm{sec}$. After the last cycle, a final extension was performed at $72^{\circ} \mathrm{C}$ for $10 \mathrm{~min}$, and the RT-PCR products were separated by electrophoresis on $1.5 \%$ agarose gels.

Real-time quantitative qPCR of CENP-H cDNA was carried out (Bio-Rad Laboratories, Hercules, CA, USA) with SYBR $^{\circledR}$ Priemix Ex Taq ${ }^{\mathrm{TM}}$ II (Takara Biotechnology). The cycling conditions were as follows: initial denaturation at $95^{\circ} \mathrm{C}$ for $30 \mathrm{sec}$, and then 40 cycles of denaturation at $95^{\circ} \mathrm{C}$ for $5 \mathrm{sec}$, annealing and elongation at $60^{\circ} \mathrm{C}$ for $30 \mathrm{sec}$. Bio-Rad CFX Manager 2.1 software was used for analysis of qPCR. The 
Table II. Associations between CENP-H protein expression and the clinicopathological features of HCC cases.

\begin{tabular}{|c|c|c|c|c|}
\hline \multirow[b]{2}{*}{ Characteristics } & \multirow[b]{2}{*}{$\begin{array}{l}\text { Cases } \\
\mathrm{N}=60\end{array}$} & \multicolumn{2}{|c|}{$\begin{array}{l}\text { CENP-H-positive } \\
\text { cases, n }(\%)\end{array}$} & \multirow[b]{2}{*}{ P-value } \\
\hline & & $\begin{array}{l}\text { Low } \\
(33)\end{array}$ & $\begin{array}{l}\text { High } \\
(27)\end{array}$ & \\
\hline Age (years) & & & & 1.000 \\
\hline$>60$ & 9 & $5(55.6)$ & $4(44.4)$ & \\
\hline$\leq 60$ & 51 & $28(54.9)$ & $23(45.1)$ & \\
\hline Gender & & & & 0.519 \\
\hline Male & 49 & $26(53.1)$ & $23(46.9)$ & \\
\hline Female & 11 & 7 (63.6) & $4(36.4)$ & \\
\hline Histopathological grade & & & & 0.001 \\
\hline I-II & 37 & $27(73.0)$ & $10(27.0)$ & \\
\hline III-IV & 23 & $6(26.1)$ & $17(73.9)$ & \\
\hline TNM clinical stage & & & & 0.002 \\
\hline $\mathrm{I}-\mathrm{II}$ & 34 & $25(73.5)$ & $9(26.5)$ & \\
\hline III-IV & 26 & $8(30.8)$ & $18(69.2)$ & \\
\hline Lymph node metastasis & & & & 0.739 \\
\hline Negative & 49 & $26(53.1)$ & $23(46.9)$ & \\
\hline Positive & 11 & $7(63.6)$ & $4(36.4)$ & \\
\hline Distant metastasis & & & & 0.085 \\
\hline Negative & 57 & $33(57.9)$ & $24(42.1)$ & \\
\hline Positive & 3 & $0(0)$ & $3(100)$ & \\
\hline Tumor size $(\mathrm{cm})$ & & & & 0.032 \\
\hline$\leq 5$ & 23 & $17(73.9)$ & $6(26.1)$ & \\
\hline$>5$ & 37 & $16(43.2)$ & $21(56.8)$ & \\
\hline Venous invasion & & & & 0.129 \\
\hline Negative & 29 & $19(65.5)$ & $10(34.5)$ & \\
\hline Positive & 31 & $14(45.2)$ & $17(54.8)$ & \\
\hline Chinese clinical stage & & & & 0.008 \\
\hline I & 12 & $9(75)$ & $3(25)$ & \\
\hline II & 29 & $19(65.5)$ & $10(34.5)$ & \\
\hline III & 19 & $5(26.3)$ & $14(73.7)$ & \\
\hline
\end{tabular}

housekeeping gene GAPDH was used as an internal control for both RT-PCR and real-time qPCR. The optimization and synthesis of primers was carried out by Sangon Biotech Co., Inc. (Shanghai, China). Serial dilutions of the template cDNA were made for reactions to optimize the PCR products within the linear range.

Protein extraction and western blotting. Frozen tissue samples were first solubilized $1 \mathrm{~h}$ in lysis buffer (Beyotime Institute of Biotechnology, Shanghai, China) in ice using homogenizers, then centrifugated (14,000 x g; Eppendorf, Germany) $30 \mathrm{~min}$ at $4^{\circ} \mathrm{C}$. After denaturation, equal amounts of supernatant proteins were separated electrophoretically on $10 \%$ SDS/polyacrylamide gels (SDS-PAGE) and transferred onto polyvinylidene difluoride membranes (Millipore, Bedford, MA, USA) in a tank transfer apparatus (Bio-Rad). The membranes were blocked with 5\% skim milk in Tris-buffered saline with Tween (TBS-T) for $2 \mathrm{~h}$, then incubated with mouse anti-CENP-H antibody diluted 1:200 (Santa Cruz Biotechnology, Santa Cruz, CA, USA) overnight at $4^{\circ} \mathrm{C}$. The next day, horseradish peroxidase-conjugated anti-mouse immunoglobulin G (HRP; Santa Cruz Biotechnology) diluted 1:5,000 in phosphate-buffered saline (PBS pH 7.4) was used as the secondary antibody. Antigens on the membrane were detected with enhanced chemiluminescence horseradish peroxidase (HRP) substrate (Millipore) according to the manufacturer's instructions. The intensity of each band was measured using Image Lab 4.0 (Bio-Rad). To confirm equal loading, mouse anti- $\beta$-actin antibody (Santa Cruz Biotechnology) diluted 1:1,000 was used as the primary antibodies.

Immunohistochemistry. CENP-H expression was detected by immunohistochemistry through a standardized streptavidinperoxidase (SP) method. For immunohistochemistry, $4-\mu \mathrm{m}$ paraffin sections were adhered to glass slides, which then were deparaffinized in fresh xylene and rehydrated by passage through an ethanol series. Following antigen retrieval in citrate buffer $(0.01 \mathrm{M}, \mathrm{pH} 6.0)$ in a pressure cooker for 1-2 min after air jetting, slides were first cooled to room temperate and washed by distilled water and PBS twice successively, then incubated for $10 \mathrm{~min}$ with $0.3 \%(\mathrm{v} / \mathrm{v})$ hydrogen peroxide to block activity of endogenous peroxidase. After blockage with $10 \%$ normal goat serum for $10 \mathrm{~min}$, the slides were incubated with mouse monoclonal anti-CENP-H (1:40 dilution; Santa Cruz Biotechnology) overnight at $4^{\circ} \mathrm{C}$. The next day, the slides were washed in PBS three times and incubated with biotinylated goat anti-mouse IgG (1:100 dilution; Beijing Zhongshan Golden Bridge Biotechnology Co., Ltd., Beijing, China) as the secondary antibodies for $20 \mathrm{~min}$. After being washed with PBS and incubated with peroxidase-conjugated streptavidin (1:100 dilution; Beijing Zhongshan Golden Bridge Biotechnology) for $10 \mathrm{~min}$, each slide was colored with $100 \mu 10.02 \%$ 3,3'-diaminobenzidine (DAB) (Sigma, St. Louis, MO, USA). Finally, all the paraffin sections were rinsed by running water to terminate coloration, counterstained with hematoxylin, differentiated through $0.1 \%$ hydrochloric acid $(\mathrm{HCl})$, washed with PBS, dehydrated in graded ethanol and coverslipped with neutral gum. All the incubations were carried out in a humidified chamber, and non-immune mouse serum replaced the primary antibody as the negative control. The slides were read and scored by two independent experiments under a microscope (Olympus Optical Co., Ltd., Tokyo, Japan). According to Guo et al (21), the extent of staining and the proportion of stained cells were used as criteria of evaluation. First, according to the intensity of staining, the cells were scored on a scale of 1, no staining; 2 , weak staining (light yellow); 3 , moderate staining (yellowish brown); 4 , strong staining (brown). Second, according to the percentage of positively stained tumor cells, the proportion of CENP-H-positive cells varied from 0 to $100 \%$ : $<5 \%$ of the cells, $1 ; 6-35 \%$ of the cells, $2 ; 36-70 \%$ of the cells, $3 ;>71 \%$ of the cells, 4 . If the final score, calculated by multiplying the above two scores, was $\geq 4$, the tumor was considered to have high expression; otherwise, the tumor was considered to have low expression. 
A

B
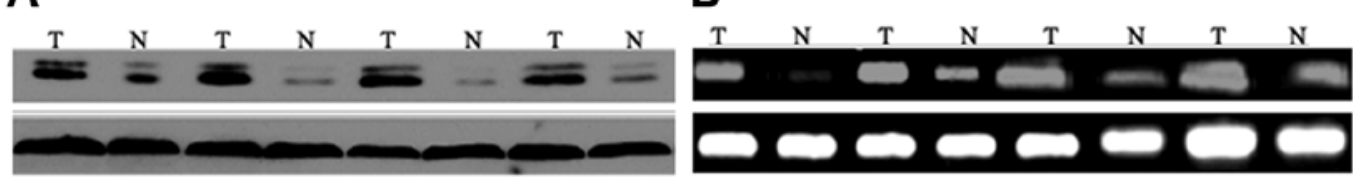

C

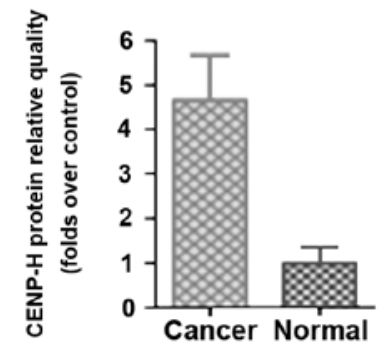

D

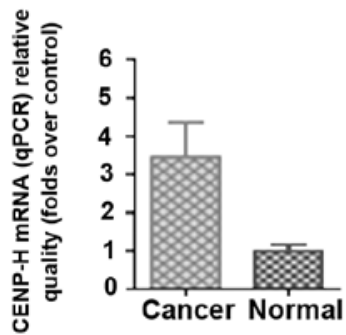

Figure 1. CENP-H mRNA and protein expression in HCC and the corresponding adjacent non-cancerous tissues. (A) Representative western blot analysis of the CENP-H protein product in 10 matched independent HCC and the corresponding adjacent non-cancerous tissues. (B) Representative RT-PCR analysis of the above 10 matched samples. (C) Relative quality of the total CENP-H protein in the 10 samples. (D) The total CENP-H mRNA (qPCR) relative quality in the 10 matched samples.

Cell culture. The liver cancer cell line Hep3B obtained from the Transform Medical Center of Xi'an Jiaotong University was cultured in Dulbecco's modified Eagle's medium (DMEM) (Invitrogen) supplemented with $10 \%$ fetal bovine serum (HyClone, Logan, UT, USA) and $1 \%$ penicillin/streptomycin, and incubated at $37^{\circ} \mathrm{C}$ in a humidified $5 \% \mathrm{CO}_{2}$ atmosphere.

Immunocytofluorescence staining. To observe the expression and localization of CENP-H in Hep3B cells, $1.6 \times 10^{5}$ cells were fixed by acetone, and incubated with mouse monoclonal anti-CENP-H primary antibody (1:40 dilution; Santa Cruz Biotechnology) overnight after antigen retrieval and blockage, followed by fluorescein isothiocyanate (FITC), conjugated rabbit anti-mouse IgG (1:100 dilution; Beijing Zhongshan Golden Bridge Biotechnology). 4,6-Diamidino-2-phenylindole (DAPI) (Sigma) was used to stain the nucleus. Fluorescent imaging was observed with a fluorescence microscope (Leica QFISH; Leica Microsystems, Tokyo, Japan).

Statistical analysis. All statistical analyses were carried out using SPSS 19.0 statistical software (SPSS, Inc., Chicago, IL, USA). CENP-H protein and mRNA levels were determined by the t-test. Chi-square and Fisher's exact tests were used to analyze the relationship between CENP-H protein expression and the clinicopathological characteristics. Survival rate was calculated by the Kaplan-Meier method, and differences were examined by the log-rank test. Factors found to be associated with survival rate were then selected for a stepwise Cox's multivariate proportional hazard model to determine their prognostic values. $\mathrm{P}<0.05$ was considered to indicate a statistically significant result.

\section{Results}

Upregulation of CENP-H mRNA and protein levels in HCC tissues. To investigate whether CENP-H is upregulated in the HCC tissue, western blot analysis was performed in 10 matched pairs of HCC tissues and corresponding non-cancerous tissues from the same patient. The result revealed that CENP-H was highly upregulated in all 10 pairs of HCCs (Fig. 1A). The relative expression of CENP-H protein in tumor samples compared with adjacent non-cancerous samples varied from 1.75 to 13.13 .

To determine whether upregulation of CENP-H is the result of increased transcription, CENP-H mRNA levels in $\mathrm{HCC}$ tissues and corresponding non-cancerous tissues were examined using RT-PCR and real-time qPCR. All HCC tissues showed higher expression of CENP-H mRNA when compared with that in non-cancerous tissues (Fig. 1C). Therefore, relative mRNA levels in tumor samples correlated well with the relative protein levels (Fig. $1 \mathrm{~B}$ and $\mathrm{C}$ ). These results revealed that CENP-H was upregulated at both the mRNA and protein levels in clinical HCC tissues and that the overexpression of CENP-H may occur at the transcription level.

Immunohistochemistry of CENP-H in HCC samples and non-cancerous samples. To further study the expression and subcellular location of CENP-H protein, immunohistochemical analysis was performed in 60 pairs of paraffin-embedded HCC samples and corresponding adjacent non-cancerous samples. We observed that CENP-H expression was significantly higher in HCC tissues (38/60, 63.3\%; Fig. 2B-D) than that in the corresponding adjacent non-cancerous tissues (21/60, 35\%; Fig. 2A) ( $\mathrm{P}=0.003)$. Moreover, the expression of CENP-H appeared to increase with histological grade of HCC; well-differentiated HCC samples had the lowest expression of CENP-H (Fig. 2B), and moderately differentiated samples had higher expression (Fig. 2C), while the expression of poorly differentiated samples was the highest (Fig. 2D). CENP-H was predominantly localized in the nucleus.

Relationship of CENP-H expression with clinicopathological features. After we performed immunohistochemistry, we investigated the correlation between the expression of CENP-H protein and the clinicopathological features in $60 \mathrm{HCC}$ cases. As shown in Table II, statistical analysis revealed no statistical 


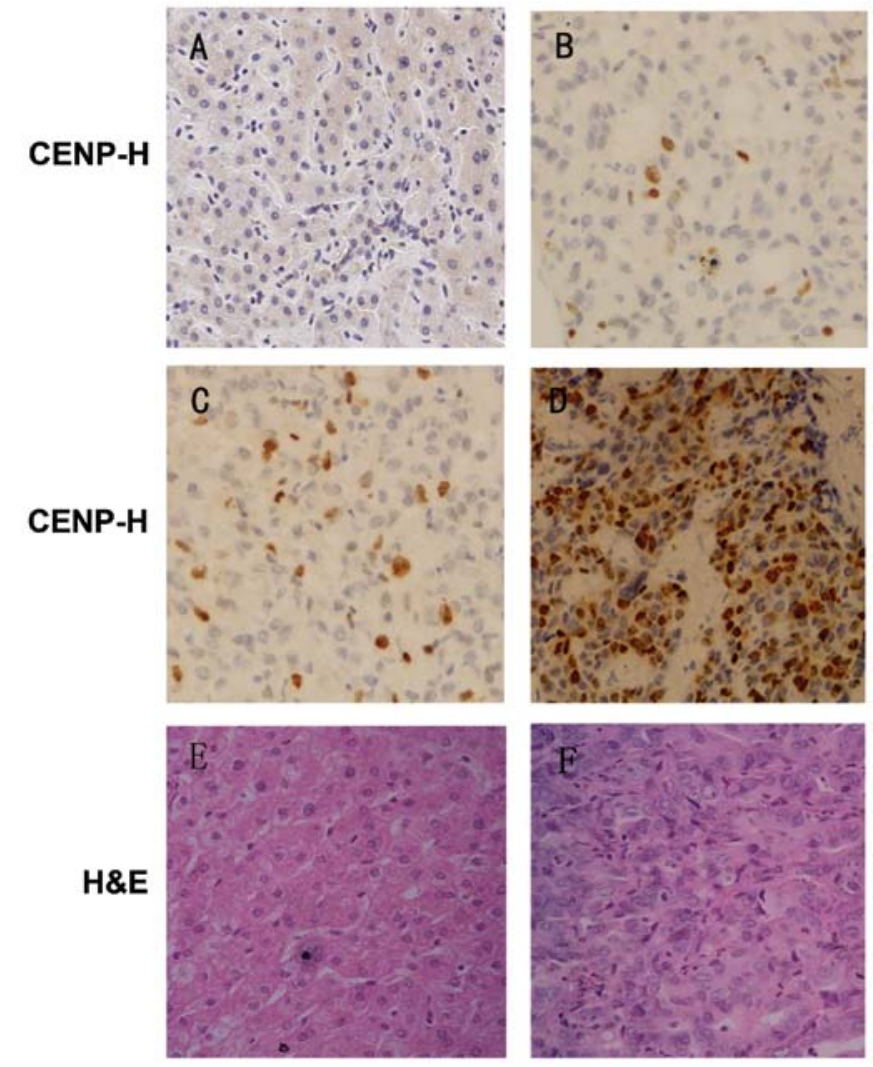

Figure 2. Representative immunohistochemistry results of CENP-H expression in $\mathrm{HCC}$ and the corresponding adjacent non-cancerous tissues. Paraffin sections were immunostained as described in Materials and methods. (A) Non-cancerous liver tissues, (B) well-differentiated HCC tissues, (C) moderately differentiated $\mathrm{HCC}$ tissues, (D) poorly differentiated HCC tissues. (E) H\&E staining of non-cancerous liver tissues, (F) H\&E staining of HCC tissues. The CENP-H staining was predominantly nuclear in the samples examined.

correlation between the CENP-H protein level and age, gender, lymph node metastasis, distant metastasis and venous invasion. However, the expression of CENP-H protein was closely related to histological grade, with higher histological grade associated with a higher frequency of CENP-H overexpression in HCC patients $(\mathrm{P}=0.001)$. In addition, statistics showed a significant difference in CENP-H expression in patients categorized according to tumor size $(\mathrm{P}=0.032)$. Therefore, expression of CENP-H protein was statistically correlated to both TNM stage $(\mathrm{P}=0.002)$ and Chinese $(\mathrm{P}=0.008)$ clinical stage.

Expression and localization of CENP-H protein in Hep $3 B$ cells. To study the expression and localization of CENP-H protein in HCC cells, Hep3B cells were stained with anti-human CENP-H monoclonal antibody using an immunofluorescence assay. Consistent with the results of the immunohistochemical analysis CENP-H was present in the nucleus of Hep3B cells (Fig. 3).

Survival analysis. To determine the effect of classic clinicopathological characteristics and CENP-H expression on survival in HCC, we analyzed the cumulative survival of patients through Kaplan-Meier analysis (Fig. 4). A log-rank test showed that the low CENP-H expression group had longer survival time, whereas the high CENP-H expression group was associated with shorter survival $(\mathrm{P}<0.05)$.
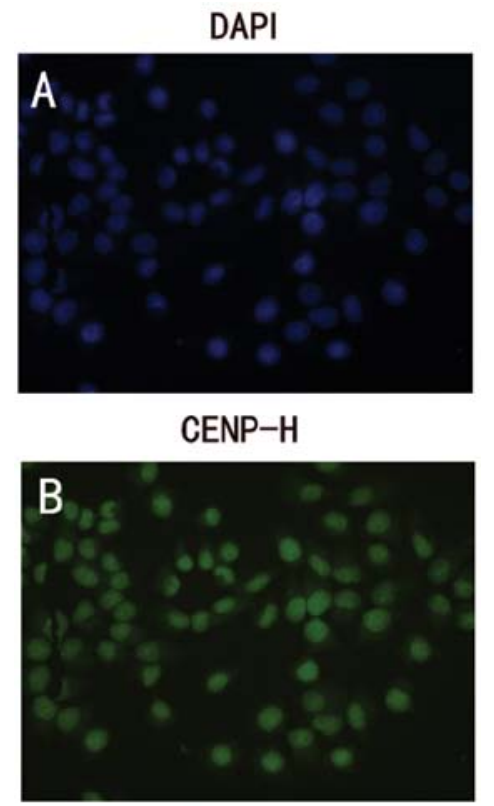

Merge

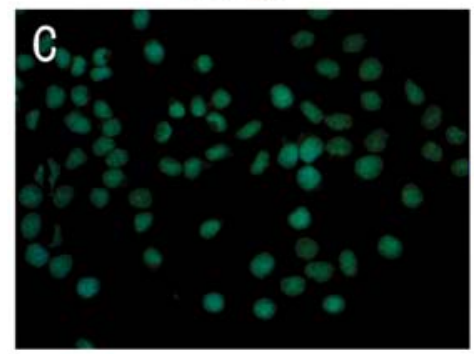

Figure 3. Representative immunofluorescence analysis of CENP-H expression in Hep3B cells. CENP-H nuclear expression in Hep3B cells. (A) DAPI was used to stain nucleus; (B) the immunofluorescence staining of CENP-H protein; (C) merging of images $\mathrm{A}$ and $\mathrm{B}$, which showed CENP-H nuclear expression in Hep3B cells.

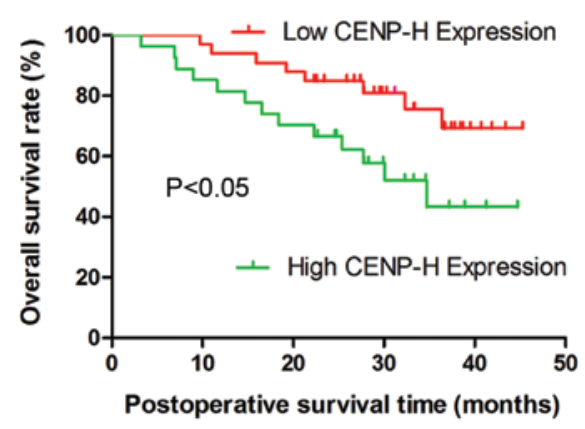

Figure 4. Kaplan-Meier analysis of the overall postoperative survival curves in HCC patients according to their immunohistochemical staining as low or high of CENP-H protein expression. Result showed that the low CENP-H expression group had longer survival time, whereas the high CENP-H expression was associated with shorter survival $(\mathrm{P}<0.05)$.

Multivariate analysis in our study revealed that venous invasion $(\mathrm{P}=0.037)$, advanced TNM stage $(\mathrm{P}=0.026)$ and Chinese stage $(\mathrm{P}=0.020)$ were independent prognostic factors for overall survival time in patients with HCC. Overexpression of CENP-H protein was also an independent prognostic factor for overall survival $(\mathrm{P}=0.046)$. Tumor size and other clinical parameters were not independent prognostic factors. 


\section{Discussion}

HCC is characterized by rapid growth, early metastasis, a high recurrence rate and poor patient prognosis $(4,5,7)$. The present study was designed to detect the expression of CENP-H in HCC samples to determine its clinical significance for HCC patients and to better define its potential role as a prognostic factor. CENP-H, located on the inner centromere plate, has been found to play a fundamental role in the organization and function of the active human centromere complex $(17,18)$. In the present study, we first revealed the differential expression of CENP-H at both the mRNA and protein levels in HCC tissues and adjacent non-cancerous tissues. In addition, upregulation of the CENP-H protein level was consistent with the change in the corresponding mRNA level, indicating that the overexpression occurs at the transcriptional level. The results of the immunohistochemical analysis also confirmed the overexpression of CENP-H protein in the HCC tissues, consistent with the results of the western blotting. The present study suggests that high CENP-H expression is closely correlated with worse histological grade, advanced TNM and Chinese clinical stage, and larger tumor size. In contrast, the Chi-square and Fisher's exact test did not indicate a positive correlation between CENP-H expression and age, gender, lymph node metastasis, distant metastasis or venous invasion. Regarding the localization of CENP-H, immunohistochemistry of paraffin-embedded tissues and immunofluorescence of Hep3B cells showed nuclear staining, which is consistent with other reports $(19,25)$.

The equal distribution of genetic material to the two daughter cells is the ultimate goal of mitotic cell division, which is ensured by the correct assembly of kinetochore at each centromere locus $(28,29)$. Deregulation of centromere proteins is believed to be related to aneuploidy and carcinogenesis $(10,30)$. CENP-A has been shown to be overexpressed and mistargeted in human colon cancer cells (31), which can lead to kinetochore malfunction (14). Li et al (32) demonstrated that CENP-A was upregulated in HCC, the overexpression of which prompted HCC cell proliferation, while the depletion of CENP-A inhibited HCC cell growth and induced apoptosis. In addition, CENP-A has been associated with cancers such as breast cancer and lung adenocarcinoma $(33,34)$. At the same time, CENP-E, CENP-F, and inner centromere protein have been demonstrated to contribute to carcinogenesis (35-38). All observations suggest that deregulation of centromere proteins is common in the development and progression of various tumors.

CENP-H has been shown to be overexpressed in several types of tumors. Tomonaga et al (25) found that CENP-H was upregulated in both primary colorectal cancer tissues and CIN cell lines and that overexpression induces aneuploidy and interphase micronuclei, a characteristic of chromosome missegregation. CENP-H is also confirmed to be a prognostic marker for nasopharyngeal carcinoma, tongue cancer, esophageal carcinoma, and human non-small cell lung cancer (19-22). Fukagawa et al (17) discovered that, in the absence of CENP-H, chicken DT40 cells were arrested in metaphase and died of chromosome missegregation. Similarly, Orthaus et al (24) observed that knockdown of CENP-H in human HEp-2 cells induced aberrant mitotic phenotypes and decreased the number of living cells. Similarly, the present study found CENP-H to be upregulated in $\mathrm{HCC}$ and its overexpression was correlated with the overall survival rate. All these findings illustrate that CENP-H plays an important role in tumorigenesis and progression.

Centrosome aberration can induce genetic instability in HCC (39). CENP-A, another inner centromere protein, has been observed to be amplified in HCC (32). Similarly, we found that CENP-H was overexpressed in HCC tissues. According to Tomonaga et al (25), the deregulation of CENP-H prevented it from localizing to the centromere by depleting the factors that recruit it to the centromere. As a consequence, functional CENP-H protein decreased, and the normal kinotechore assembly was disrupted. Meanwhile, depletion of functional CENP-H reduces hBubR1 activation through mislocalizing kinetochore-associated microtubule motor protein CENP-E (24), which stimulates the kinase activity of hBubR1 and is required for the establishment and maintenance of the checkpoint in mitosis. Defects in checkpoints are closely associated with tumorigenesis $(15,29)$. All of these findings provide the theoretical basis that overexpression of CENP-H results in defects in checkpoints and CIN and eventually plays a key role in the development and progression of HCC. However, further studies are needed to investigate the specific mechanisms of overexpression of CENP-H in HCC and how it contributes to tumorigenesis and development.

In summary, CENP-H is upregulated in $\mathrm{HCC}$ and is closely related to larger tumor size, worse histological grade and advanced TNM and Chinese stages. Although the mechanisms require further elucidation, the overexpression of CENP-H may be used as a novel biomarker for HCC prognosis.

\section{Acknowledgements}

We thank the staff of the Transform Medical Center, Xi'an Jiaotong University, for their technical assistance.

\section{References}

1. Siegel R, Naishadham D and Jemal A: Cancer statistics, 2013. CA Cancer J Clin 63: 11-30, 2013.

2. Jemal A, Bray F, Center MM, et al: Global cancer statistics. CA Cancer J Clin 61: 69-90, 2011.

3. Altekruse SF, McGlynn KA and Reichman ME: Hepatocellular carcinoma incidence, mortality, and survival trends in the United States from 1975 to 2005. J Clin Oncol 27: 1485-1491, 2009.

4. Khorsandi SE and Heaton N: Contemporary strategies in the management of hepatocellular carcinoma. HPB Surg 2012: $154056,2012$.

5. Llovet JM, Fuster J and Bruix J: Intention-to-treat analysis of surgical treatment for early hepatocellular carcinoma: resection versus transplantation. Hepatology 30: 1434-1440, 1999.

6.Franco D, Capussotti L, Smadja C, et al: Resection of hepatocellular carcinomas. Results in 72 European patients with cirrhosis. Gastroenterology 98: 733-738, 1990.

7. Schwartz M, Roayaie S and Konstadoulakis M: Strategies for the management of hepatocellular carcinoma. Nat Clin Pract Oncol 4: 424-432, 2007.

8. Rajagopalan $\mathrm{H}$ and Lengauer C: Aneuploidy and cancer. Nature 432: 338-341, 2004.

9. Ricke RM and van Deursen JM: Aneuploidy in health, disease, and aging. J Cell Biol 201: 11-21, 2013.

10. Cimini D and Degrassi F: Aneuploidy: a matter of bad connections. Trends Cell Biol 15: 442-451, 2005.

11. Bakhoum SF and Compton DA: Chromosomal instability and cancer: a complex relationship with therapeutic potential. J Clin Invest 122: 1138-1143, 2012. 
12. Liang QJ, Lu XF, Cheng XL, et al: The active expression of $\mathrm{CenpB}$, a constitutive protein in the centromeres of chromosomes, in breast cancer tissues. Yi Chuan Xue Bao 31: 236-240, 2004 (In Chinese).

13. Sugata N, Munekata E and Todokoro K: Characterization of a novel kinetochore protein, CENP-H. J Biol Chem 274 27343-27346, 1999.

14. Yuen KW, Montpetit B and Hieter P: The kinetochore and cancer: what's the connection? Curr Opin Cell Biol 17: 576-582, 2005.

15. Bakhoum SF and Compton DA: Kinetochores and disease: keeping microtubule dynamics in check! Curr Opin Cell Biol 24 64-70, 2012.

16. Kramer A, Neben $\mathrm{K}$ and Ho A: Centrosome replication, genomic instability and cancer. Leukemia 16: 767-775, 2002.

17. Fukagawa T, Mikami Y, Nishihashi A, et al: CENP-H, a constitutive centromere component, is required for centromere targeting of CENP-C in vertebrate cells. EMBO J 20: 4603-4617, 2001.

18. Sugata N, Li S, Earnshaw WC, et al: Human CENP-H multimers colocalize with CENP-A and CENP-C at active centromere kinetochore complexes. Hum Mol Genet 9: 2919-2926, 2000.

19. Liao WT, Wang X, Xu LH, et al: Centromere protein $\mathrm{H}$ is a nove prognostic marker for human nonsmall cell lung cancer progression and overall patient survival. Cancer 115: 1507-1517, 2009.

20. Liao WT, Song LB, Zhang HZ, et al: Centromere protein $\mathrm{H}$ is a novel prognostic marker for nasopharyngeal carcinoma progression and overall patient survival. Clin Cancer Res 13: 508-514, 2007.

21. Guo XZ, Zhang G, Wang JY, et al: Prognostic relevance of Centromere protein $\mathrm{H}$ expression in esophageal carcinoma. BMC Cancer 8: 233, 2008.

22. Liao WT, Yu CP, Wu DH, et al: Upregulation of CENP-H in tongue cancer correlates with poor prognosis and progression. J Exp Clin Cancer Res 28: 74, 2009.

23. Shigeishi $\mathrm{H}$, Higashikawa $\mathrm{K}$, Ono $\mathrm{S}$, et al: Increased expression of CENP-H gene in human oral squamous cell carcinomas harboring high-proliferative activity. Oncol Rep 16: 1071-1075, 2006.

24. Orthaus S, Ohndorf S and Diekmann S: RNAi knockdown of human kinetochore protein CENP-H. Biochem Biophys Res Commun 348: 36-46, 2006.

25. Tomonaga $\mathrm{T}$, Matsushita $\mathrm{K}$ and Ishibashi $\mathrm{M}$ : Centromere protein $\mathrm{H}$ is up-regulated in primary human colorectal cancer and its overexpression induces aneuploidy. Cancer Res 65: 4683-4689, 2005.

26. Hamilton SR and Aaltonen LA: World Health Organization Classification of Tumors: Pathology and Genetics of Tumours of the Digestive System. LARC Press, Lyon, 2000.
27. Sobin LH and Fleming ID: TNM Classification of Malignant Tumors, fifth edition (1997). Union Internationale Contrele Cancer and the American Joint Committee on Cancer. Cancer 80: 1803-1804, 1997.

28. Cleveland DW, Mao Y and Sullivan KF: Centromeres and kinetochores: from epigenetics to mitotic checkpoint signaling. Cell 112: 407-421, 2003.

29. Schmidt JC, Arthanari H and Boeszoermenyi A: The kinetochore-bound Skal complex tracks depolymerizing microtubules and binds to curved protofilaments. Dev Cell 23: 968-980, 2012.

30. Kops GJ, Weaver BA and Cleveland DW: On the road to cancer: aneuploidy and the mitotic checkpoint. Nat Rev Cancer 5: 773-785, 2005

31. Tomonaga T, Matsushita K, Yamaguchi S, et al: Overexpression and mistargeting of centromere protein-A in human primary colorectal cancer. Cancer Res 63: 3511-3516, 2003.

32. Li Y, Zhu Z, Zhang S, et al: ShRNA-targeted centromere protein A inhibits hepatocellular carcinoma growth. PLoS One 6: e17794, 2011

33. McGovern SL, Qi Y, Pusztai L, et al: Centromere protein-A, an essential centromere protein, is a prognostic marker for relapse in estrogen receptor-positive breast cancer. Breast Cancer Res 14: R72, 2012.

34. Wu Q, Qian YM, Zhao XL, et al: Expression and prognostic significance of centromere protein $\mathrm{A}$ in human lung adenocarcinoma. Lung Cancer 77: 407-414, 2012.

35. Wood KW, Lad L, Luo L, et al: Antitumor activity of an allosteric inhibitor of centromere-associated protein-E. Proc Natl Acad Sci USA 107: 5839-5844, 2010.

36. O'Brien SL, Fagan A, Fox EJ, et al: CENP-F expression is associated with poor prognosis and chromosomal instability in patients with primary breast cancer. Int J Cancer 120: 1434-1443, 2007.

37. Shigeishi H, Mizuta K, Higashikawa K, et al: Correlation of CENP-F gene expression with tumor-proliferating activity in human salivary gland tumors. Oral Oncol 41: 716-722, 2005.

38. Barbanis S, Ioannou M, Kouvaras E, et al: INCENP (inner centromere protein) is overexpressed in high grade non-Hodgkin B-cell lymphomas. Pathol Oncol Res 15: 11-17, 2009.

39. Nakajima T, Moriguchi M, Mitsumoto Y, et al: Centrosome aberration accompanied with p53 mutation can induce genetic instability in hepatocellular carcinoma. Mod Pathol 17: 722-727, 2004. 